\title{
Passive sensing on mobile devices to improve mental health services with adolescent and young mothers in low-resource settings: the role of families in feasibility and acceptability
}

\section{Sujen Man Maharjan}

Transcultural Psychosocial Organization Nepal

Anubhuti Poudyal

George Washington University https://orcid.org/0000-0002-6426-2646

Alastair van Heerden

Human Sciences Research Council https://orcid.org/0000-0003-2530-6885

\section{Prabin Byanjankar}

Transcultural Psychosocial Organization Nepal https://orcid.org/0000-0001-9517-2881

\section{Ada Thapa}

George Washington University

Celia Islam

George Washington University

Brandon A Kohrt ( $\nabla$ bkohrt@gwu.edu )

https://orcid.org/0000-0002-3829-4820

\section{Ashley Hagaman}

Yale University https://orcid.org/0000-0002-8016-1036

Research article

Keywords: Adolescent, Child Health, Depression, Developing Countries, Digital Health, Digital Phenotype, Mental Health, Postpartum Depression, Psychotherapy, Nepal

Posted Date: November 4th, 2020

DOl: https://doi.org/10.21203/rs.3.rs-22755/v2

License: (9) (1) This work is licensed under a Creative Commons Attribution 4.0 International License. Read Full License 
Version of Record: A version of this preprint was published at BMC Medical Informatics and Decision Making on April 7th, 2021. See the published version at https://doi.org/10.1186/s12911-021-01473-2. 


\section{Abstract}

Background: Passive sensor data from mobile devices can shed light on daily activities, social behavior, and maternal-child interactions to improve maternal and child health services including mental healthcare. We assessed feasibility and acceptability of the Sensing Technologies for Maternal Depression Treatment in Low Resource Settings (StandStrong) platform. The StandStrong passive data collection was piloted with adolescent and young mothers, including mothers experiencing postpartum depression, in Nepal.

Methods: Mothers (15-25 years old) with infants ( $<12$ months old) were recruited in person from vaccination clinics in rural Nepal. They were provided with an Android smartphone and a Bluetooth beacon to collect data in four domains: the mother's location using the Global Positioning System (GPS), physical activity using the phone's accelerometer, auditory environment using episodic audio recording on the phone, and mother-infant proximity measured with the Bluetooth beacon attached to the infant's clothing. Feasibility and acceptability were evaluated based on the amount of passive sensing data collected compared to the total amount that could be collected in a 2-week period. Endline qualitative interviews $(n=31)$ were conducted to understand mothers' experiences and perceptions of passive data collection.

Results: 782 women were approached and 320 met eligibility criteria. 38 mothers ( 11 depressed, 27 nondepressed) were enrolled. Of 9,605 possible readings per sensor, 5,579 audio recordings [mean $(M)=57.4 \%$; median $(M d n)=62.6 \%], 5,001$ activity readings $(M=50.6 \% ; M d n=63.2 \%), 4,168$ proximity readings ( $M=41.1 \% ; M d n=47.6 \%)$, and 3,482 GPS readings $(M=35.4 \% ; M d n=39.2 \%)$ were obtained. Feasibility challenges were phone battery charging, data usage exceeding pre-paid limits, and burden of carrying mobile phones. Acceptability challenges were privacy concerns and lack of family involvement. Overall, families' understanding of passive sensing and families' awareness of potential benefits to mothers and infants were the major modifiable factors to increase acceptability and reduce gaps in data collection.

Conclusion: Approximately half of all possible passive data readings were collected. Feasibility challenges can be addressed by providing alternative phone charging options, setting up reverse billing for the app, and exploring smartwatches as a replacement for mobile phones. Enhancing acceptability will require greater family involvement and improved communication regarding benefits of passive sensing for psychological interventions and other health services.

Registration: International Registered Report Identifier (IRRID): DERR1-10.2196/14734

\section{Background}

Passive sensing on mobile devices refers to the capture of information that does not require users' active input while they go about their daily lives [1,2]. For example, accelerometers on smartphones can detect activities such as walking, riding in a vehicle, and standing, and the Global Positioning System (GPS) 
captures location. Passive sensors also provide information on the number of steps taken in a day, heart rate variability, exposure to light and sound, and proximity to others with mobile devices. Passive sensing data provides a window onto experiences, behavior, and environments of individuals, all of which are important to understand mental health and mental illness.

Because the field of mental health lacks objective markers of disease such as viral loads, pathogen detection, and point-of-care testing for disease status, passive sensing provides a unique objective reference for mental health status $[3,4]$. There are a number of initiatives to explore potential benefits from using passive sensing data in mental health and behavioral health studies. Passive sensing data was collected with people with lived experience of mental health challenges in Australia [5]. Passive sensing has been used to identify mood instability [6]. Passive sensing has recorded time away from home and activity levels to identify risk of early dementia [7]. Other studies have similarly explored the potential of using passive sensing data in depression [3, 8], bipolar disorder [9], and schizophrenia [10] using GPS, audio, accelerometer, and Bluetooth devices.

Passive sensing data collection can be especially helpful for health initiatives in low- and middle-income countries (LMIC), which are characterized by limited access to specialty health services and where some populations have low literacy [11]. Combined with effective interventions, passive sensing data collection has the potential to address major public mental health issues in LMIC.

One mental illness of high prevalence and societal impact is postpartum depression, particularly because young mothers are often not identified or treated in LMIC. The prevalence of postpartum depression in LMIC ranges from $3 \%$ to $32 \%$ [12]. Passive sensing can help to better understand maternal mental health by recording physical activity, location, sleep, mother-child interaction, and the auditory environment. In a high-income country study of women with elevated perinatal depression symptoms, radius travelled as measured with GPS was associated with depressed mood. Women who traveled larger radii had milder depression than the women with severe depression who had smaller travel radii [13]. Given that maternal mental illness is associated with disrupted sleep, lack of social engagement, lack of stability in daily schedules, and altered interaction patterns with their infants [14-16], there is a host of opportunities to apply passive sensing data collection to improving diagnosis, monitoring, and treatment for mothers with depression.

Passive sensing data collection also has the potential to be a more accurate and less burdensome approach to detection when compared with traditional paper-based screening tools. Typically, self-report checklists-in either paper-based or electronic formats-are used for identification of mothers with depression in low resource settings. Because specialists are often unavailable, tools such as the Edinburgh Postnatal Depression Scale (EPDS), Patient Health Questionnaire (PHQ-2 and PHQ-9) become de-facto diagnostic tools [17]. These tools typically have high false positive rates (low specificity), which can add further burden to health systems trying to deliver mental health services [18]. To additionally complicate the situation, these tools need considerable cultural adaptation to perform appropriately with diverse global populations [19-22]. 
This strategy of passive sensing using personal mobile phones is a low-cost alternative to identifying mothers with depression because it does not rely solely upon hiring health workers to conduct clinicbased or community screenings. Because an increasing number of mothers now have a smartphone, even in low-resource settings, there are not high costs for additional technology [23]. It is also not a time burden for mothers, which would be required for completing assessments. Passive sensing does not have the same social desirability bias introduced when responding to a health worker administering a screening tool. Passive sensing is also a real-time process which can provide tailored feedback to both mothers and health workers, as opposed to screening tools that have potential recall bias and are overview of experience over the past one to two weeks. Furthermore, passive sensing may have greater sensitivity (fewer false positives) by identifying those persons with an objective impairment in social, behavioral, and physical activities. Passive sensing also illuminates in which domains a person has impairment, whereas psychological symptoms on screening questionnaires may not reveal this information.

Before designing a passive sensing-informed intervention program, it is crucial to understand the feasibility of collecting data [24] and cultural factors influencing acceptability [25]. Mobile technology for health (mHealth) use in the real world is impacted by technical challenges, familiarity with technologies, and cultural attitudes and practices [26]. Therefore, in this study, we explore the feasibility, acceptability, and perceived utility of collecting passive sensing data among depressed and non-depressed adolescent and young mothers in rural Nepal. We pilot test the passive sensing data collection component of the Sensing Technologies for Maternal Depression Treatment in Low Resource Settings (StandStrong) platform. Specifically, through a smartphone app and Bluetooth Low Energy beacon, sensing data are passively collected on mothers' geographic movement, physical activity, the audio environment, and mother-infant proximity. The two main sources of data are the amount of passive sensing data recorded and qualitative interviews with mothers about their experience of the passive data collection process.

\section{Methods}

\section{Overview}

The study protocol is outlined in detail elsewhere [27]: International Registered Report Identifier (IRRID): DERR1-10.2196/14734. The procedures and results described here refer to Component 2 of the original study protocol. For details on the study according to the EHEALTH extension to CONSORT guidelines [28] see attached Supplemental File 1 and RE-AIM framework [29] in Supplemental File 2. Recruitment and data collection occurred between November 2018 through April 2019.

In brief, young mothers (15-25 years of age) with infants ( $<12$ months of age) were recruited from vaccination clinics in rural Nepal. Both depressed and non-depressed young mothers were recruited. The mothers then participated in 2 weeks of passive sensing data collection capturing her physical activity, geographic movement, the auditory environment, and mother's proximity to her infant. Technologies 
piloted for passive sensing included Android smartphones, smartwatches, and Bluetooth Low Energy beacons.

\section{Setting}

The study was conducted in a setting that exemplifies health resources. This site was Chitwan district, a southern region of Nepal. The total population of Chitwan is 579,984 . The under 5 mortality rates for Chitwan is 38.6 per 1000 (national average is 52.9 ); it also has a higher literacy rate of $78.9 \%$ compared to the national average of $67 \%$ [30]. Chitwan district was selected because of a longstanding established partnership with the local health system and a district-wide scaling-up of community-based mental health services that was being conducted [31].

\section{Study Population and Sampling}

Study participants were young mothers ( $15-25$ years old) with infants ( $<12$ months), including both mothers with and without postpartum depression. Recruitment of mothers was conducted at infant immunization camps held as seven health facilities in rural areas of Chitwan. Camps were typically attended by 136 mothers on average every month. Inclusion criteria were mothers between 15-25 years of age with an infant aged between 1-12 months living in the study area, and willingness to be screened for postnatal depression. There were no inclusion requirements for computer/internet literacy on the part of mothers because of the passive nature of the mobile sensing data collection. The intention was to assess the feasibility among representative mothers in the community, which includes women with limited technology literacy.

To determine eligibility, trained research assistants approached mothers at immunization to ask their age and infant's age, after which they conducted the consent procedures. For mothers 15-17 years old, assent was obtained, and a guardian provided consent. Because of passive sensing data collection that captured information about the household. A meeting was held with the mother's household representative to describe the study and data collection procedures. If mothers and their family members agreed to the study, passive sensing data were collected for 2 weeks (14 consecutive days; details on passive sensing described below).

The age range of 15-25 years was selected because this is based on the United Nations definition of youth which includes 15-24 years of age [32]. Similarly, the lower age limit of 15 years old was used because data are routinely collected on pregnancy for mothers 15 years of age and above, such as is in the Nepal Demographic Health Survey (DHS). UNICEF adolescent reporting is divided in young adolescents (10-14 years old) and older adolescents (15-19) in the Multiple Indicator Cluster Surveys (MICS) reporting. We included 25 years of age as well because of prior research on suicide deaths in Nepal which showed the greatest burden of suicide mortality among women was $\leq 25$ years of age [33]. 
A sample size calculation was not conducted because this is a pilot study and the recruitment was done based on feasibility of using these devices by the participants [34,35]. Our goal was to recruit 25 depressed and 25 non-depressed mothers. To allow for potential dropouts, we allowed for recruitment of up to 27 consenting mothers in each category. We enrolled more mothers than our target because we anticipated dropouts due to novelty of the study and potential reluctance from the participants in using the technology. This sample was based on feasibility of the number of mothers in the youth age range attending local clinics during the study period.

\section{Mental Health Measure}

Mothers depression status was determined with a locally validated Nepali version of the Patient Health Questionnaire (PHQ-9), which is 9-item self-report tool for depression screening widely used in both highincome countries and LMIC [19]. We categorized the mothers as "depressed" and "non-depressed" based on their PHQ-9 total score. Mothers scoring below 9 were classified as 'non-depressed,' and those above 9 as 'depressed'. Among Nepali adults presenting to outpatient services, a cut-off of 9 has a sensitivity of $94 \%$ and specificity of $69 \%$, positive predictive value (PPV) of 0.33 and negative predictive value (NPV) of 0.99. A cut-off of 7 has a sensitivity of 1.00 , specificity of 0.55 , PPV of 0.26 , and NPV of 1.00 . Of note, psychometric values specific for Nepali postpartum mothers aged 15-25 are not available. Additional details on study measures are available in the published protocol [27].

\section{Technology and passive sensor data collection}

Passive sensing was collected through two devices: mothers were given a low-cost Android Samsung J2 Ace smartphone and a Bluetooth Low Energy beacon to be attached to her infant's clothing. The devices used in this study were selected following extensive ethnographic inquiry regarding acceptability and feasibility in the study site [25]. The two devices selected for this study (smartphone and Bluetooth beacon) were considered culturally acceptable and feasible. The Samsung J2 Ace smartphone is a costeffective mobile phone (US $\$ 160$ ) that is popular in the study setting. Most common, low-end mobile phones in Nepal cost US $\$ 70-\$ 120$, and therefore the device selected in the study was slightly more expensive than commonly-used devices. In the study area, most individuals owned mobile phones or have family members who own mobile phones. Hence, there is less risk of stigmatization because of phone use in the study. We selected the Samsung J2 Ace phone because it is widely available for purchase within Nepal and it was the cheapest option that could effectively run all the features and apps required for the study.

For a subset of mothers, we also piloted the use of smartwatches in place of the smartphone. Models included the Zeblaze Thor 4 Android smartwatch and Lemfo Lem8 Android smartwatch. The cost of the smartwatches was approximately $\$ 200$. The smartwatches are not yet commonly used compared to the mobiles but it has the potential of providing the same data in a more convenient device. We gave it only to the subset of the mothers as we did not have extensive experience in testing the smartwatches with the 
mothers but we got the feedback from the Community Advisory Board (CAB) that this option should also be explored as it could be good alternative to mobiles reducing interference in their daily lives.

The Bluetooth Low Energy beacon was the RadBeacon dot (\$10-15) developed by Radius Networks [36]. We used a closed cloth pouch (Nepali: thaili) to hold the beacon around the baby's waist and prevent the infant from being able to remove or play with the device. Because the beacon was sewn into the pouch around the waist, the baby could not access the beacons, or get hold of the device. The research assistants informed the mothers about the safe use of beacons, including caution while using the device, so the device would not cause physical discomfort to the baby. The use of the RadBeacon Dot was approved by the Nepal Health Research Council for the purpose of this study. RadBeacon Dot also has United States Federal Communications Commission (FCC) certification. FCC is a body that oversees the permissible exposure level for all devices with radio frequency. In the United States, the Food and Drug Administration (FDA) relies on FCC for inputs on medical devices [73]. According to FDA, devices such as activity trackers are general wellness devices because these devices have "(1) an intended use that relates to maintaining or encouraging a general state of health or a healthy activity or (2) an intended use that relates the role of healthy lifestyle with helping to reduce the risk or impact of certain chronic diseases or conditions and where it is well understood and accepted that healthy lifestyle choices may play an important role in health outcomes for the disease or condition" [74]. Additionally, in our study, RadBeacon is not a medical device used for treatment or for the transmission of health information (e.g., temperature, pulse, respiration) from the infant. It is only used to track proximity between mother and infant during daytime hours. Regarding the safety of exposure for infants, the FCC limit for radiation from devices is $1600 \mathrm{~mW} / \mathrm{kg}$ [75], which equates to approximately $800 \mathrm{~mW}$ for a $5 \mathrm{~kg}$ infant. The RadBeacon Dot specifications are +4 to $-20 \mathrm{dBm}$, which equates to 2.5 to $0.1 \mathrm{~mW}$. These ranges are comparable to an infant in a house with a standard wireless network and Bluetooth devices.

The mothers were provided with the phone and the beacon for the duration of the study. They returned the devices after completing data collection. The smart devices collected 4 types of data-proximity, episodic audio, physical activity, and geographic location. To collect these data, we installed our custombuilt Electronic Behavior Monitoring app (EBM version 2.0). The EBM app passively collected data for 30 seconds every 15 min between 4AM and 9PM. A folder, NAMASTE, was created automatically once the EBM app was downloaded on the smartphone (see EBM screenshots in Figure 1). All data were stored in the folder. Because all sensing and data collection was done passively, mothers did not need to interact with the app in any way to enable data collection. Details of each passive sensing domain are provided below:

1. Proximity of mother to infant: The proximity sensor (Bluetooth Low Energy beacon) was fitted to the infant's clothing, and the mother was asked to carry the mobile phone to record when the mother was in proximity to the infant. Every 15 minutes, the EBM app scanned for the presence of advertising packets from the assigned Bluetooth beacon and recorded whether the beacon was present or not. Through the proximity beacon, we calculated the daily interaction routine between mother and child. Routinization of daily behavior is associated with positive mood, less fatigue, and lower risk of 
maternal depression $[54,55]$. We hypothesized that non-depressed mothers will have a more consistent routine than depressed mothers over the 2-week period. Additionally, we anticipated depressed mothers to have less self-care time, and less social support to take care of the baby. Lack of social support is correlated to risk and severity of postpartum depression $[53,59,60]$, while high levels of instrumental social support are associated with lower postpartum depression symptom severity [61].

2. Episodic audio recording: For episodic audio recording conducted approximately every 15 minutes, the microphone in the phone was used to record 30-second audio clips saved in an MP3 format. The audio data were saved in a local folder on the device before being uploaded to the cloud and processed. We analyzed the audio data by categorizing audio clips as "speech" and "non-speech" sounds. We compared speech and non-speech sounds for depressed and non-depressed mothers to predict differences based on the exposure to human speech. Depressed mothers are more likely to have prolonged periods without verbal communication compared with nondepressed mothers. Human speech sounds as a proxy for social interaction helps us measure social isolation which is associated with postpartum depression [64]. Also, lack of social group membership is also considered a risk of postpartum depression [65] especially for adolescent mothers. Limited verbal engagement between mothers and infants is also a manifestation of postnatal depression and predicts poor development for children [66,67].

3. Physical activity: Activity recognition was used to record the predicted activity type (e.g., walking, standing still, cycling, riding a vehicle) at the time of audio recording based on the mobile phone's accelerometer data. In this study, we used the accelerometer and gyroscope sensors along with the Android Activity Recognition API, which is built on top of these sensors. The Activity Recognition API automatically detects activities such as walking, running, riding in a vehicle, or standing still. Selfreported physical limitations are correlated with postpartum depression severity [53]. Wrist actigraphy measurements among postpartum women showed an association between disrupted routines and poor mental health outcomes; postpartum women with dysrhythmic fatigue patterns reported more stress and less vigor compared with the women where fatigue patterns followed consistent daily cycles [55].

4. Geographic location: GPS on the mobile phone collected the mother's position each time the phone had an activity event (phone unlocking, Bluetooth scanning etc.). We included GPS domain as studies have shown association between GPS radii and depression. In a study of pregnant mothers, the daily radius of travel was associated with depression symptoms, with greater depression levels associated with more restricted radii of travel [57]. An increase in depressive symptoms predicted smaller radii of travel in subsequent days.

We had previously produced a video to explain these data collection processes to potential study participants [25]. In addition, confidentiality management, such as deleting audio files was piloted with mothers using similar devices in South Africa [37]. On the devices, mothers can delete audio files at any time. They could also ask the research assistants to not transfer the data if they did not wish to share it with the team. Mothers were also instructed to turn off their phone anytime they chose in order to stop 
data collection. Because of our prior work piloting passive sensing technologies and evaluating mothers' ability to delete data $[25,37]$, we did not conduct further formal usability testing with mothers for this activity within the context of the currently described study.

A female research assistant briefed each mother and her family on the technical use of the phone and beacon. All research assistants self-identified as employees of the NGO Transcultural Psychosocial Organization Nepal. The research assistant visited the mother's home on average 3-5 times including for study briefing and consent, day one of data collection for technology delivery and training, day three of data collection for technology troubleshooting, and then weekly, with intermittent phone calls to additionally troubleshoot and provide any needed technology support. Mothers were instructed to keep their mobile phones with them as much as possible and attach the beacon to their infant's clothing throughout the day. Mothers were asked to turn the mobile phones off and remove the beacon from the child during the night. Mothers' identifiable information (name, phone numbers) were stored in a secured server. The app was password protected, with counselors needing passcode to access the app. All the passive sensing data and qualitative data were stored under a unique participant code (without identifying information) and stored in a secured cloud-based server. Mothers and family members had the opportunity to become comfortable with the researcher assistants because of the repeated home visits by research staff.

No prompts or reminders were provided to mothers electronically once the EBM was installed. Research assistants visiting the home would check functioning of EBM app and detection of the Bluetooth beacon then conduct trouble shooting as needed. Once the mother was comfortable sharing the data, research assistants copied the data from the phones in a portable device. The data did not contain any identifiable information, and contained passive sensing data in .csv and $\mathrm{mp} 4$ format. The data folder was coded with a de-identified ID to anonymize the data. It was then uploaded to a secure cloud server, through a secured connection and removed from the local devices immediately (within 24 hours). The research team did not listen to the audio data manually, but used machine learning to process the data and generate predictions on the sounds.

Two types of app errors were collected namely, app exceptions and user engagement issues. App exceptions and failures can have multiple causes. Commonly they include errors in code logic which can be introduced, for example, when code runs on different versions of Android, or hardware interfaces are implemented in a non-standard way by device manufacturers. User engagement issues include trouble remembering a password, trying to perform an unsupported function, and/or struggling to find the function they are looking for. In this study we tracked fatal app exceptions and user-login challenges.

\section{Qualitative data collection}

To assess feasibility and acceptability of passive data collection, we triangulated several sources of data including in-depth interviews (IDIs) performed at the end of 14 days of passive sensing data collection, field notes recorded by research assistants from each participant encounter, and memos documenting 
the significant events related to experience of technology use documented by the a project lead based in the Chitwan study site. Female research assistants conducted IDIs using a semi-structured interview guide lasting between 20-45 minutes. Questions elicited maternal experiences and perceptions of the technology and EBM application, covering feasibility, social acceptability, confidentiality, utility, and recommendations for improvement. Our inquiry focused on confidentiality and social acceptability given important ethical considerations of passive data collection. Questions regarding these domains were elicited both from the mother as well as from her family throughout the study period. Importantly, the research assistant had established meaningful rapport with both the mother and her family (on average visiting the mother's home 3-5 times), permitting more comfort and allowing detailed and frequent field notes to capture examples and texture not captured by the IDI, as well as notes related to confidentiality concerns from either the mother or her family members. Qualitative interviews were audio-taped, transcribed, and translated before coding and analysis. The interviews were first transcribed verbatim in Nepali and then translated to English by a bilingual translator. We followed a standardized Nepali mental health glossary for translation of emotional and psychological terms into English [38]. The Consolidate Criteria for Reporting Qualitative Studies (COREQ) checklist is included in Supplemental File 3 [39].

\section{Data analysis}

\section{Passive sensing data analysis}

The proximity, activity, and GPS were captured and stored in the comma separated file (Flat file) and the audios were captured as .mp4 audio file. Thus, the captured audio files were further processed through TensorFlow/Al/Machine learning system to predict the information contained in the audio with the outputs of the predictions made in comma separated files. Raw data retrieved from the sensors using the EBM v2.0 app was saved on the device with one file being produced per day. The time stamped data were then extracted from these csv files before being processed and loaded into a SQL database. The same approach was followed for each of the study devices resulting in a single aggregated data set per sensor, i.e. proximity, activity, audio, and GPS. During this process raw proximity data were turned into an hourly binary reading indicating the presence or absence of a beacon. Raw activity data were trimmed from the full list of activities detected along with their probability scores to only the activity with the highest associated probability. The 30 seconds audio wav files were processed by our machine learning model and the audio classification label with the highest probability score saved. The application of Convoluted neural networks to machine vision now allow object detection to be performed by machines at a level almost on par with humans [40]. While acoustic scene classification is not as mature, the field is rapidly advancing [41]. For this project we used a model built around the AudioSet dataset which includes labeled examples of 527 sounds e.g., thunderstorms, dogs barking and people speaking. We used this general audio classifier to process our 30 second audio clips and extract the most likely (if any) sound present in the recording. Finally, GPS data was also dichotomized indicating either the presence or absence of a GPS reading with an accuracy reading of less than 50 meters. 
These four datasets were then loaded into Python where they were merged into a single data frame with a datetime index. Whenever a sensor had no hourly reading it was coded as missing. This data frame was then cleaned to remove data of mothers who dropped out of the study, and fix outliers introduced by some readings missing a valid datetime value. Exploratory data analysis was performed on this data frame including the calculation of measures of central tendency, range and missing value analysis. Plots and tables were produced on the full dataset (4AM to 9PM), a dataset trimmed to include only values between 7AM and 7PM and finally a dataset trimmed to include only values between 6AM and 9PM.

\section{Qualitative data analysis}

In our prior formative study, we categorized feasibility and acceptability of wearable technology for passive sensing data collection for health research in LMIC into 6 domains [25]. We incorporated these for the current study, exploring domains relevant to our data including: technical issues, interference, confidentiality, safety, utility, and communication. Interview transcripts were combined with their relevant field notes, providing additional context. Three researchers (AH, AP, SM) independently read two transcripts each to generate common themes. SM generated a preliminary codebook. AH, AP, and SM then modified and iterated the codebook during the process of obtaining intercoder agreement. Intercoder agreement was assessed between all coders on a portion of coded data until a kappa of 0.76 was achieved. Three researchers (SM, AT, $\mathrm{Cl}$ ) coded the interview transcripts in NVivo 12 [42]. Code summaries were produced for each of the sub-domains following an applied thematic approach [43, 44]. Summaries were discussed, enhanced, and revised with several authors (SM, AT, AP, AH) to ensure depth, breadth, and systematic comparisons across informants. Case memos were independently reviewed and analyzed by SM and AT to identify case studies relevant to the domains of the paper. We did not approach the participants later for interviews who dropped out of the study but we prepared detailed dropout case memos to record the reasons for dropout and related significant information.

\section{Ethics and participant safety}

The study was approved by the Nepal Health Research Council (\#327/2018) and George Washington University's Institutional Review Board (\#051845). As a compensation for their time contribution in the study, the mothers received a set of clothes and toys for their children (US\$3-5). The implementing research organization, Transcultural Psychosocial Organization (TPO) Nepal, had an institutional suicide protocol in place for handling and reporting any kind of adverse event during the study including suicide. During screening and assessments, we did an in-depth risk evaluation and the counselor provided immediate support if there was any risk of suicide. In case of emergency, we had set up a referral system with the government hospital for the immediate care by the psychiatrist.

\section{Results}




\section{Recruitment and Sample}

We screened 782 mothers, of whom 320 were eligible for inclusion based on age criteria of the mothers and infants. We consented the eligible mothers and screened them for depression. Approximately $92 \%$ of the eligible mothers scored below the depression cut-off $(n=294)$ and $8 \%$ scored above the cut-off $(n=26)$. We serially enrolled non-depressed mothers. Approximately two-thirds of the non-depressed mothers and their families provided consent and were enrolled, with a final sample of 27 non-depressed mothers consenting to participate in the passive data collection. All mothers subsequently screened who scored below the depression cut-off were not eligible for the study. Of the 26 mothers who screened positive for depression, 11 of the mothers and their families provided consent and enrolled in the study. Table 1 contains demographic information of the participants. Case memos revealed reasons for nonparticipation of both non-depressed and depressed mothers, and memos were also used to document reasons for withdrawing from the study. Reasons for not participating included mothers moving outside of the study area, inability of the research team to contact mothers following initial screening in the health facilities, families not-consenting to participate in the study, and mothers too busy to participate due to family obligations. Two participants withdrew from the study before completing the full 2 weeks of passive sensing data collection (additional details on why they withdrew are provided below).

\section{Passive Sensing Data collection}

We collected activity, audio, GPS and infant proximity data through passive sensor readings (every 15 minutes from 4 am to 9pm, daily). Table 2 provides the total passive data collected between 4 AM - 9 PM among depressed, non-depressed, and the total sample. Audio and activity data were captured more often than GPS and proximity data on average. For the 38 young mothers, the cumulative maximum number of passive sensor readings (every 15 minutes from 4am to 9pm, daily) was 9,605, see Table 2 . This total of 9,605 was obtained because data collection was for two weeks (14 days) for 18 hours a day for 38 moms $(38 * 18 * 14=9,576)$. Another 29 readings resulted from some participants with atypical start times of their passive sensing collection or delayed cessation of their collection based on when research assistants could meet them.

Per participants, there were, on average, $57.4 \%$ of audio, $50.6 \%$ of activity, $41.1 \%$ of proximity, and $35.4 \%$ of GPS data readings. Figure 2 shows the average passive data readings by the time of the day. Data collection was lower than the total possible readings in the early morning across all sensors and tapered off at night, but was generally consistent from 10 AM to 6 PM. We explored possible explanations for differences in successful data collection by time of day and sensor type along with description of qualitative results to illuminate these differences.

Four issues emerged during the implementation phase of this pilot that were fixed and rolled out in subsequent releases of the app. The issues were: 
- The proximity chart was not displaying correctly on the J2Ace phone Samsung A7 tablet and the graphing software had to be updated.

- Users requested that the proximity chart display order be reversed.

- Added a daily routine award card

- Fixed an issue with the Nepali Date Calendar which was not displaying the correct Gregorian date/time when converted.

User engagement was tracked and $30 \%$ of app usage time was spent in the participant details page viewing data, awards, and posts. Another $20 \%$ of time was spent reading the posts (after clicking on the summary in the participant details page). Scrolling, filtering, and searching made up the other $50 \%$ of time spent in the app. The 4-digit pin password used was entered correctly on the first try in $94 \%$ of login attempts.

We categorize these findings below based on the 6 qualitative domains for feasibility and acceptability (see Table 3).

Mobile phone battery charge, data usage, and positive and negative family involvement were the main technical feasibility issues identified that limited data collection throughout the day. Passive sensing requires the phone to be turned on. Morning data (and some evening data) were most likely to be missing because mothers typically were instructed to turn their phones off at night before bed and then turn it back on when awakening in the morning. Subsequently, there were gaps in data collection in the evening and early morning hours.

"I used to switch off this watch at night and connect to the charger and switch on in the morning. ... In the daytime I used to switch off this watch again and connect it to the charger."

- (19-year old depressed mother)

Common feasibility challenges likely reduced data capture across all sensor collection. These feasibility challenges included lack of electricity, mothers forgetting to charge the phone, devices not retaining charge (especially smart watches), technical difficulties such as phones not connecting to the charger properly, or environmental factors such as protecting the device from rain. In general, there were more technical issues reported by mothers who used smartwatches with frequent battery draining. We tested the use of smartwatches instead of mobile phones with three participants but $0 \%$ of the activity data was collected due to the smartwatch not supporting activity data collection (see Text Box 1). The use of smartwatches with no activity data contributed to reduce the average across all participants to $60 \%$ at best, with the greatest data capture during midday. 


\section{Text Box 1: Limitations of passive sensing data collection with a smartwatch}

For a 21-year old non-depressed mother with a 4-month old infant, it was her first experience of using a smartwatch. She did not have a problem attaching the beacon on the child's clothing. However, she felt uncomfortable wearing the watch on her wrist. This was largely due to her workload at home -she washed clothes and did dishes multiple times a day, which necessitated her to get her hands and arms wet. She was concerned about possible water damage to the watch. She also had trouble keeping the watch charged because it lost battery quickly. Her husband helped her complete the study, providing reminders and assistance charging the watch. She eventually completed the study with her husband's support who helped her charge the smartwatch. We collected $62.5 \%$ and $51.9 \%$ audio and proximity data but were unable to collect GPS or activity data due to limited functionality of the smartwatch.

Of the total possible readings, about $47 \%$ of the GPS data was collected during the high period of data capture from 7AM - 7 PM, see online Supplemental File 4). A likely reason for less successful data capture compared to audio was that GPS data collection was not triggered the same way as audio, proximity, or activity data were. The GPS data collection required WiFi or $3 \mathrm{G}$ signal for collection. Therefore, data collection was low because mothers were mostly indoors which limited this GPS connection. Initially, we used a combined method that included both mobile data and direct GPS connection to collect GPS location of the mothers. However, mothers and their families quickly used available data, typically by watching YouTube videos, which then led to not enough prepaid data for GPS collection. We switched to direct connection to the satellite to address the loss of data due to low mobile data by turning on high accuracy in the GPS settings.

\section{Domain 2: Interference}

We explored daily interference related to mobile phone and Bluetooth beacon use. Consistent daily mobile phone use was acceptable to most mothers. However, carrying two mobile phones (one for the study and one personal phone) was considered interfering with daily activities. Additionally, mothers shared their concern about the probability of losing mobile phones if they had to carry two phones at all times. Mothers working in a shop or office outside the home and mothers who were farming found it even more challenging to carry two mobile phones. Additionally, it was difficult for the study team to contact mothers who worked during the day if she had technical issues with the devices, which caused further disruption in data collection [Text Box 2].

\section{Text Box 2: Working mothers and passive data collection}

A 23-year old non-depressed mother with a 10-month old infant was a small business owner of a shop in which she made toy dolls and trained others in this trade. She received assistance from her family to use the smartwatch. Her husband helped her charge the watch when the battery was low, reminded her to wear the watch, and put the beacon on the child (in the secured pouch). As she was busy with her business's work, it was difficult for her to have enough free time for regular study team visits. So, she suggested recruiting housewives instead of employed mothers so that they could dedicate their time to the study. She completed the study, and we were able to collect $62.7 \%$ of audio and $54.1 \%$ of proximity data. (No GPS or activity data were recorded due to technical issues in the smartwatch.) 
The participants who did not find mobile phones interfering typically wore clothes with pockets or used the mobile phones for non-study related activities such as watching videos or listening to music.

Bluetooth beacons presented more challenges with daily interference. This may have been due to beacon novelty to the mothers so they felt more concerned initially about the device than about mobile phones. One of the mother's major concerns was physical discomfort to the baby. Mothers generally put the beacons on the baby and took it off at night, or during oil massages and baths. Some mothers found the routine of putting beacons on the baby tedious after the first few days with some mothers delaying or forgetting to put the beacon on the baby after bathing or oil massages.

The proximity data was consistently lower than both audio and activity data with about $60 \%$ data collection at midday, even though it was triggered at the same time as audio and activity. One possible reason could be the participants turned off the Bluetooth signal on their mobile phones.

\section{Domain 3: Confidentiality}

We collected about $75 \%$ of audio data during the daytime hours of 7 AM to 7 PM (median value for all participants, see Supplemental File 4). Audio recordings were the data type most concerning to the participants from a confidentiality perspective. At least 11 mothers in our study expressed concerns of being audio recorded. One of the reasons was fear that study staff would listen to the audio clips and share private information with other people in the community, or that the community would know about the family disputes. For instance, two participants reported:

"If I talk about difficulty, mostly I am worried that other people will listen to all our discussions. They will know all our family problems. And they will talk about our problems everywhere."

- (24-year old depressed mother)

Interviewer [I]: Do you like this beacon and this mobile? Do you like to use this mobile and fix this beacon on your baby?

Participant [P]: I like using the devices but I feel worried because you might know all our family matters and our conversations.

I: We don't listen to those recordings.

P: You will not share those recordings with other people?

- (21-year old depressed mother)

Some participants reported changing their behavior such as spending more time with the baby, talking lovingly or softly around the baby, shutting the mobile off during family arguments, or asking family members to not use bad words. Each of these behaviors may have reduced or biased the audio capture. Mothers with family members that abused alcohol and mothers in conflict with their mothers-in-law 
usually expressed the greatest privacy concerns. Family members of such households also asked mothers to switch their mobile phones off when they were under the influence of alcohol, or delete audio recordings that had their voices (See Textbox 3 ).

"We have different conditions in our home. Sometimes people quarrel and we have arguments in our house. This mobile might record all those things so I have to switch off this mobile."

- (17-year old non-depressed mother)

P: [Smiling] This mobile records sound. And all the recordings were stored in its memory so my husband told me to delete his recordings.

I: When did you switch off this mobile at night?

P: Sometimes at 6 or sometimes at $7 \mathrm{pm}$.

I: And what about your sisters and your own mother?

P: Sometimes when I start talking about my mother-in-law, my mother tells me not to talk about her, this mobile might record the voice and save it.

- (16-year old non-depressed mother)

\section{Text Box 3: Deletion of data and other reasons for low data capture}

The family of a 21-year old depressed mother lived in a temporary squatter settlement without electricity g near the jungle. They agreed to participate, but due to lack of electricity they charged their mobile phones at a neighbor's house. The participant was concerned that the device may get stolen and worried about needing to cover its expenses. The study team provided her with a power bank to charge the mobile and assured her and her family not to worry if something happened to the device, there would be no financial consequence. The provision of a power bank helped to keep the mobile running for a longer period of time.

We collected $73.9 \%$ of activity, $41.6 \%$ of audio, $10.5 \%$ of GPS, and $29.0 \%$ of proximity data from the mother. The low GPS data collection was a result of excessive data usage. During data collection, we relied only on mobile data to collect GPS. This and other similar situations where mothers ran out of prepaid data prompted us to change the connectivity so that the phones had direct connection to the satellite. There was lower audio and proximity data collection in comparison to her activity data. In our qualitative interviews, the mother shared that her husband and mother-in-law listened to the audio files and deleted the ones that had their voices. Another reason for her relatively low data capture was that the proximity data collection was interrupted when the Bluetooth was turned off on the phone, which was another reason for the low data collection. The participant's husband used to turn off the Bluetooth and keep the mobile for his own entertainment purposes due to which proximity data was interrupted.

\section{Domain 4: Safety concerns}

Three types of safety concerns were highlighted by participants: (1) safety of the infant when the Bluetooth beacon was attached to his/her clothing, (2) mother's safety when using mobile phones, and 
(3) physical safety of the devices. Among the three, child safety was the most concerning to the mothers. One major child safety issue was physical discomfort that the beacon could cause to the infant, such as device poking the baby during sleep.

"I think that this beacon might poke my baby and make it difficult for him to sleep."

- (21-year old non-depressed mother)

To avoid physical discomfort, mothers were instructed to remove the beacons when the baby was sleeping or mothers moved the device over multiple layers of clothes to avoid poking. The feeling of potential discomfort to the child hindered the consistent use of beacons and may have been one factor for reduced proximity data capture.

"While using these technologies, I thought this beacon might poke my baby and sometimes I removed the beacon."

- (17-year old non-depressed mother)

Despite child safety concerns, there were two major facilitators that propelled mothers to continue using the device - trust in the study staff and no adverse effect to the baby after the first few days of use. Because the study staff reached out to the mothers in health posts where mothers went for regular checkups and immunization, they trusted the health workers in the health facility. The study staff coordinated with the health workers, and were therefore looked at by the mothers as trustworthy. Second, despite initial concern, when the baby did not get sick or have adverse effects in the first few days of use, the mothers were convinced that the beacons were safe:

I: Did you think that it might affect your baby or your baby might feel difficulty due to this beacon?

P: In the beginning I had those types of [negative] thoughts but after using [the devices] regularly for many days I didn't have that type of thought anymore.

I: What types of thoughts did you have in the beginning?

P: That my baby might get sick, or it might have some health effects.

- (23-year old non-depressed mother)

Mother's safety was less of a concern in comparison to child's safety. In general, all the mothers thought the mobile phones and beacons did not have an adverse effect on the mothers. A factor facilitating the use of smartphones was mother's prior experience with mobile phones. Because mothers were familiar with smartphones, they did not think it would affect their health.

The final safety concern we explored was potential theft or breakage of the devices. Mothers, especially the ones from poor economic backgrounds, were scared that the study devices could get stolen or broken. 
Despite assurance from the study team that they were not liable in case of theft or accident, mothers were still anxious, especially for the first few days. The study team provided support and reassurance to the mothers during subsequent home visits to assuage remaining anxiety related to device safety.

\section{Domain 5: Perceived utility}

In general, mothers and families did agree to and continue using the phones throughout the period because of perceived benefits. Some of the perceived benefits aligned with the study goals, while some benefits were non-study related. Among the study-aligned perceived utility, mothers mentioned using the beacon and mobile phone to know the distance between them and their babies throughout the days. They could see the actual distance in smart devices. They also knew the mobile recorded their sounds, movement, and activities. Some mothers went back and listened to their audio clips. For perceived utility not related to the study purpose, mothers reported that they used the phone for listening to music, taking pictures and videos, using Facebook and watching YouTube videos. Perceived utility, however, varied across participants. For example, when asked about the utility of the beacons, some mothers said that the study showed how much they loved their babies. Other participants speculated that the data could help understand growth and brain development of the baby, or help in conflict resolution at home.

I: - I gave you this watch and fixed this beacon on your baby for two weeks. How was your experience these two weeks? What was your experience while using this watch and beacon? Please share something about that.

P: - I think I got a chance to provide more care to my baby. I got a chance to learn many things from the technologies that you provided me. This watch helps to find out whether we are speaking the truth or not. This watch records our voices continuously for a long time. One thought is continuously stuck in my heart-mind: through these recordings we can find out if someone is hiding something.

- (21-year old non-depressed mother)

Some mothers thought the technology could record the time spent with the baby or identify their mood changes during the day.

I: - Do you know anything about why you are using this beacon and mobile? Though I had already told you about its use, what do you think about this technology?

P: - These technologies are for observing the changes in a mother like being irritated, distance between mother and the baby and problems in family relations. In the future, our daughter-in-law's granddaughters would benefit from this technology. That's why I agreed to use this technology.

- (24-year old depressed mother)

Domain 6: Communication 
Communication facilitated passive data collection through three subdomains - study team engagement, tech literacy, and autonomy of using devices. The importance of the study team engagement was critical when explaining the technology and addressing any queries that mothers had during the study duration. Mothers enjoyed their interactions with the study staff, especially when the study staff asked them about their children and family. They enjoyed study staff visiting them every few days to discuss any new queries and talk to the family members about the technology. We also provided the mothers with a study brief handout in Nepali, as a support tool, so that other family members and neighbors could read and understand about the study themselves during and after the consent process. The study tool supported mothers in answering family's or neighbors' questions about the study when the study team was not physically present to answer those questions.

One of the major social influences for acceptability was collaboration with local health posts for screening and recruitment at the community level. The recommendation from the health workers helped the study team to establish rapport with the participant and then follow up through the home visits. With the study team's consistent technical support and clear communication of the study findings, the mothers felt more involved in the data collection process. They also felt more empowered to censor data collection by turning on/off the mobile device or beacon if needed. For example, 11 mothers described that they switched the phones off or left the phone in another room during family discussions, particularly to avoid recording any disputes or bad language.

Although $32 \%$ of study mothers were new to smartphone technology, all mothers confidently described their ability to navigate and operate the varying features by the second week. Mothers made decisions when or whether to attach the beacons on the child's clothing as well. To support autonomy of using the devices, we found family engagement and consent to be important facilitators in both the research process and successful implementation of passive data sensing (See Text Box 4). Mothers felt more confident and comfortable when the study team explained the technology and study objectives to their families, especially to the family members in decision making roles such as husbands and mothers-inlaw. As per our protocol, the study team visited the mother's family after the initial screening at the vaccination clinics. Family consent helped the family understand the technology better and ask questions to the study team. Mothers generally said they were able to answer questions on the study objectives independently, but the family consent helped them get support from family members when the mothers had to explain the technology to non-family members. 


\section{Text Box 4: Other reasons for low data collection-religious concerns}

No participants refused to participate because of religious beliefs with the exception of one family that was concerned that the technology was used for Christian religious conversion.

A 22-year old non-depressed mother withdrew from the study after a few days. Through follow up qualitative work, we later learned that the main reason the mother's family asked her to withdraw was that they suspected that the technological devices were being used to convert them to Christianity (due to a legacy of coercive organizations in the study region). We also learned that the participant wanted to continue the study but was forced to drop out by her husband and father-in-law.

We collected $14.5 \%$ of activity, $65.5 \%$ of audio, $6.3 \%$ of GPS, and $7.5 \%$ of proximity data from the mother. This mother was also one of the earliest participants we gave the devices to, as we were still making changes to the technology for appropriate data collection. The lower GPS data could be due to the mobile phone running out of data. Other data collection could have been affected by social factors. For example, the mother's family later told the study team that they were reluctant to use the devices, including the beacon on the child. The lower proximity data collection could mean that the Bluetooth on the mobile phone was switched off most of the time. The higher audio data indicates that the mobile phone was still switched on most of the time, although functionality such as Bluetooth was likely disabled or turned off.

\section{Discussion}

This study collected passive sensing data from 38 young mothers ( 27 non-depressed and 11 depressed) in rural Nepal. We found that approximately half to two-thirds of mothers approached provided individual and family consent. We collected activity (50.6\%), audio (57.4\%), GPS (35.4\%), and proximity $(41.1 \%)$ data from the mothers. As a point of comparison a study of passive sensing conducted in a high resource setting (Australia) with 32 people with lived experience of mental health challenges obtained $55 \%$ of possible passive scan readings [5]. Another study used $50 \%$ of possible passive sensing readings for a participant as a cut-off for analysis [3]. These studies and others using passive sensing data have encountered similar technological, privacy, and other challenges as we have described in this lowresource setting [3, 8-10,24]. In our study, positive and negative family engagement, perceived benefits of passive data collection, privacy issues, and technical limitations (such as battery and data usage) influenced the amount of passive data collected.

While the majority did not provide consent for logistical reasons, some families were unwilling to let the mothers participate, highlighting the need for careful community engaged approaches to mHealth programs. Of note, fewer mothers with depression consented and enrolled and this may have been because of difficulty or reluctance in regard to family consent because depressed mothers may have been more likely to be in families where a husband or other members abused alcohol or had other behaviors that were socially stigmatized or raised safety concerns. Previous studies have reported perceived stigma as a barrier to participation among participants with mental health conditions [45-47], which could be the case for the depressed participants in our study. Positive family involvement in the study, especially in case of severe mental health illness, has been recommended in a prior study [48].

The modest rates of initial inclusion raise concern about acceptability of the data collection and also draw attention to the perceived benefit of passive data collection. For a number of the mothers and their 
families, they were unclear about the purpose of data collection. This raises the opportunity to improve participation by providing more comprehensive explanations of how this technology will improve health services, especially when conducting exploratory studies using advanced technology in LMIC.

Families in rural areas usually have a member who lives in a different city within Nepal or outside. These families rely on mobile technology to keep in contact with their family members (in our case, mothers had husbands who were working in a different city). Such participants had used mobile devices more often, and had higher internet consumption. Mostly due to the convenience of obtaining data packages, most individuals subscribed to data packages offered by telecom companies rather than wireless service by ISPs.

Once mothers consented, their main concern and that of their families appeared to be audio recording risking loss of confidentiality. Mothers used strategies such as turning off the phone, deleting data, or leaving the phone in a different room to address these confidentiality concerns. Going forward, having the audio recordings immediately converted to visual analogs on the smartphone or scrambling audio to reduce concerns about confidentiality violations is important $[24,49,50]$. Moreover, procedures will need to be refined in the consent process to explain how confidentiality is maintained and when there are risks of confidentiality breaches. For future use of this technology, we will add more video explanations and demonstrations for the consent process so mothers and families have adequate information to understand confidentiality and make an informed decision to participate in passive sensing.

Along the lines of acceptability, our follow up study will further explore participant and family preferences, particularly the role of spouses, in passive sensing data collection, and document the challenges and opportunities at an individual- and family-level when such data collection method is used. In our study, there were instances of family members denying participation in the study, as a result of privacy concerns. We will further assess participant and family burden of employing this data collection method, and potential ways to mitigate the burden.

Technical shortfalls in data collection (e.g., limitations in data, battery, etc.) could potentially be addressed by using reverse data billing in which the research organization or health organization is billed for data collection through a post-paid contract rather than needing to provide mothers repeatedly with charge cards for data and airtime. For example, the EBM app and other StandStrong apps could be billed to the organization while charges for calls, texts, YouTube, and other personal social use are billed to the mother. In addition, in the future installing the StandStrong suite on participant's personal phones rather than giving an extra study phone could reduce the challenges related to carrying two phones and helps with the learning curve because mothers are already familiar with their own phones [51]. Use of one's own personal smartphone is common for smartphone-based interventions in high income settings.

We piloted smartwatches as another approach to passively collect data. Because smartwatches can be worn and do not require pockets or purses, we anticipated more acceptability and ease of use for mothers. However, there were a number of limitations. At this stage in smartwatch technology, we found that affordable watches had limited functionality compared to Android smartphones in the same price 
range. While the EBM app could be used in smartwatches, GPS data were collected sporadically and the accelerometer sensor did not work in smartwatches. Thus, we had to drop the smartwatches from our studies after a few participants' use. In the future, smartwatches may have increased functionality to allow for these devices to more feasibility collect the needed information compared to carrying around multiple phones $[52,53]$.

Ultimately, this study is an important first step for the integration of passive sensing data collection into health services for psychological services and other health care. We found that approximately $50 \%$ of data collection was achieved. This may be sufficient to inform some health services, and we identified a number of technical issues to improve feasibility that would increase data capture. However, the most important contribution of this work is highlighting the importance of clear and transparent communication with both young mothers and their families about the purpose and process of passive data collection. It is vital to assure that use of such technology does not increase risk of harm for mothers in vulnerable situations. Going forward additional efforts should be made to educate families about how the technology works and to have them actively engaged in the process. One possible future strategy is to explore how husbands could be more involved in the process, such as by having them also participate with the EBM app installed on their phones. Given that postpartum depression often involves relationship stressors among parents and other relatives, male involvement could both improve acceptability of passive sensing data collection and enhance the outcomes of psychological interventions.

In a subsequent study component, we will assess validity of the passive data calculating correlations across measures such as PHQ-9 and the ability of passive sensing to distinguish between depressed and non-depressed mothers. In addition, this study sets the groundwork for incorporating passive sensing data into a psychological intervention. In the research site, a psychological intervention was being delivered to support maternal mental health. The intervention, the Healthy Activity Program (HAP), is an evidence-based psychological treatment originally developed in India for delivery by lay counselors [54]. It has also shown promise in reducing the symptoms of depression in Nepal [31]. However, in HAP, counselors rely on self-report to understand a client's behavioral patterns between sessions. Passive sensing data collection visualized in the StandStrong app for counselors if integrated into HAP has the potential to provide counselors with information related to the activity patterns, sleep, social interactions, and mood of the mothers [55]. Incorporating passive data into HAP can aid counselors to provide personalized treatment. Our ultimate goal-after establishing feasibility and acceptability of passive sensing data collection as described in the current study-is to use passive sensing data collection to inform HAP delivery in Nepal [27].

\section{Limitations}

This study has a number of limitations that should qualify generalizability of the findings. The data collection strategies were evolving over the course of the study. For example, early in the study, we tried 
introducing smartwatches and then abandoned this strategy because of both limitations in which sensors worked and fears of mothers (e.g., getting the watches wet). Similarly, we changed the GPS data collection strategy midway through the study to increase data capture. Research assistants modified how they explained the study to mothers and families as we gained experience of the collection process. The reported data-capture rates mix together low collection rates from the beginning of the study and higher rates toward the end of the study. The collection rates include two participants who withdrew a few days into the collection process. Our collection rates are conservative estimates of what could be achieved in any studies going forward. Also, the technological literacy and technology landscape are rapidly changing. There is increasing availability of cellular networks in rural Nepal and rural residents are becoming more familiar with smartphones. We anticipate fewer technological barriers to this type of $\mathrm{mHealth}$ initiative in the future. However, attending to the social dynamics and daily patterns of phone use will be crucial for successful implementation of mHealth and passive data-augmented interventions in the future.

\section{Conclusion}

This study identified a number of technological barriers and facilitators to comprehensive passive data collection in a rural area of Nepal. Most of these technological barriers can be addressed. More importantly, we identified concerns related to confidentiality and interpretation of the passive sensing data collection. Passive sensing data collection has the potential to transform psychological treatments and other mental health services. Just as glucose monitoring, remote blood pressure monitoring, and other remote approaches to assessing health in real-time, real-world situations, passive sensing data can provide an as yet untapped glimpse into real world behavior and environment. However, to scale use of passive sensing data collection, the approach needs to be feasible and culturally acceptable to potential participants and their families in mHealth programs. Successful implementation of StandStrong and similar passive data collection initiatives will require addressing these concerns and fully involving family members in mHealth initiatives.

\section{Declarations}

\section{- Ethics approval and consent to participate}

Ethical approval was received from the Nepal Health Research Council (\#327/2018) and George Washington University Institutional Review Board (\#051845). We obtained written informed consent from the participants and verbal informed consent from adult members of their household.

\section{- Consent for publication}

The participants provided written consent for the publication.

\section{- Availability of data and materials}


Data will be made publicly available upon publication of the final study results.

\section{- Competing interests}

Alastair van Heerden, PhD, developed the Electronic Behavior Monitoring app (EBM version 2.0).

\section{- Funding}

The study was funded by the Bill and Melinda Gates Foundation (Grant \#. OPP1189927, PI: B.A. Kohrt). The funder played no role in the study design, analysis, or decision to publish.

\section{- Authors' contributions}

SMM, AP, and BAK drafted the manuscript. SMM, AP, AT, and $\mathrm{Cl}$ conducted the qualitative data analysis. $\mathrm{AH}$ supervised the qualitative data collection and analysis. AvH developed the EBM app. AvH and PB conducted the quantitative data analysis. SMM supervised data collection and onsite study implementation. BAK, $\mathrm{AvH}$, and $\mathrm{AH}$ conceptualized the study and study design. All authors revised the manuscript. All authors have read and approved the manuscript.

\section{- Acknowledgements}

We would like to thank Doerte Bemme for her contributions to the manuscript. We would like to thank mothers and their families who participated in this study. We also like to thank the field study team (Aasha Mahato, Bhagwati Sapkota, Sabita Lohani, Bindu Aryal, Kendra Chaudhary, Bibek KC, Sirjana Panday) and colleagues at Transcultural Psychosocial Organization Nepal.

\section{Abbreviations}

EBM

EPDS

FDA

GPS

HAP

LMIC

MICS

NDHS

NGO
Electronic Behavior Monitoring

Edinburgh Postnatal Depression Scale

Food and Drug Administration

Global Positioning System

Healthy Activity Program

Low- and Middle-Income Countries

Multiple Indicator Cluster Surveys

Nepal Demographic Health Survey

Non-Governmental Organization 
PHQ-9 Patient Health Questionnaire

PPV Positive Predictive Value

StandStrong Sensing Technologies for Maternal Depression Treatment in Low Resource Settings

TPO Transcultural Psychosocial Organization

UNICEF United Nations Children's Fund

\section{References}

1. Insel TR: Digital Phenotyping: Technology for a New Science of BehaviorDigital PhenotypingDigital Phenotyping. Jama 2017, 318(13):1215-1216.

2. Campbell AT, Eisenman SB, Lane ND, Miluzzo E, Peterson RA, Lu H, Zheng X, Musolesi M, Fodor K, Ahn G: The Rise of People-Centric Sensing. IEEE Internet Computing 2008, 12(4):12-21.

3. Saeb S, Zhang M, Karr CJ, Schueller SM, Corden ME, Kording KP, Mohr DC: Mobile Phone Sensor Correlates of Depressive Symptom Severity in Daily-Life Behavior: An Exploratory Study. J Med Internet Res 2015, 17(7):e175.

4. Bhat A, Goud BR, Pradeep JR, Jayaram G, Radhakrishnan R, Srinivasan K: Can Mobile Health Improve Depression Treatment Access and Adherence Among Rural Indian Women? A Qualitative Study. Cult Med Psychiatry 2020.

5. Boonstra TW, Nicholas J, Wong QJ, Shaw F, Townsend S, Christensen H: Using Mobile Phone Sensor Technology for Mental Health Research: Integrated Analysis to Identify Hidden Challenges and Potential Solutions. J Med Internet Res 2018, 20(7):e10131.

6. Morshed MB, Saha K, Li R, D'Mello SK, Choudhury MD, Abowd GD, Plötz T: Prediction of Mood Instability with Passive Sensing. Proc ACM Interact Mob Wearable Ubiquitous Technol 2019, 3(3):Article 75.

7. Galambos C, Skubic M, Wang S, Rantz M: Management of Dementia and Depression Utilizing InHome Passive Sensor Data. Gerontechnology 2013, 11(3):457-468.

8. Burns MN, Begale M, Duffecy J, Gergle D, Karr CJ, Giangrande E, Mohr DC: Harnessing context sensing to develop a mobile intervention for depression. J Med Internet Res 2011, 13(3):e55.

9. Beiwinkel T, Kindermann S, Maier A, Kerl C, Moock J, Barbian G, Rossler W: Using Smartphones to Monitor Bipolar Disorder Symptoms: A Pilot Study. JMIR Ment Health 2016, 3(1):e2.

10. Ben-Zeev D, Wang R, Abdullah S, Brian R, Scherer EA, Mistler LA, Hauser M, Kane JM, Campbell A, Choudhury T: Mobile Behavioral Sensing for Outpatients and Inpatients With Schizophrenia. Psychiatr Serv 2016, 67(5):558-561.

11. Ruzek JI, Yeager CM: Internet and mobile technologies: addressing the mental health of trauma survivors in less resourced communities. Glob Ment Health (Camb) 2017, 4:e16. 
12. Baron EC, Hanlon C, Mall S, Honikman S, Breuer E, Kathree T, Luitel NP, Nakku J, Lund C, Medhin G et al: Maternal mental health in primary care in five low- and middle-income countries: a situational analysis. BMC Health Services Research 2016, 16(1):53.

13. Faherty LJ, Hantsoo L, Appleby D, Sammel MD, Bennett IM, Wiebe DJ: Movement patterns in women at risk for perinatal depression: use of a mood-monitoring mobile application in pregnancy. Journal of the American Medical Informatics Association 2017, 24(4):746-753.

14. Krawczak EM, Minuzzi L, Hidalgo MP, Frey BN: Do changes in subjective sleep and biological rhythms predict worsening in postpartum depressive symptoms? A prospective study across the perinatal period. Arch Womens Ment Health 2016, 19(4):591-598.

15. Howell EA, Mora P, Leventhal H: Correlates of early postpartum depressive symptoms. Matern Child Health J 2006, 10(2):149-157.

16. Mata J, Thompson RJ, Jaeggi SM, Buschkuehl M, Jonides J, Gotlib IH: Walk on the bright side: physical activity and affect in major depressive disorder. J Abnorm Psycho/ 2012, 121(2):297-308.

17. Reynolds CF, Patel V: Screening for depression: the global mental health context. World Psychiatry 2017, 16(3):316-317.

18. Mitchell AJ, Coyne JC: Do ultra-short screening instruments accurately detect depression in primary care? A pooled analysis and meta-analysis of $\mathbf{2 2}$ studies. British Journal of General Practice 2007, 57(535):144-151.

19. Kohrt BA, Luitel NP, Acharya P, Jordans MJD: Detection of depression in low resource settings: validation of the Patient Health Questionnaire (PHQ-9) and cultural concepts of distress in Nepal. BMC Psychiatry 2016, 16(1):e58.

20. Kohrt BA, Jordans MJ, Tol WA, Luitel NP, Maharjan SM, Upadhaya N: Validation of cross-cultural child mental health and psychosocial research instruments: adapting the Depression Self-Rating Scale and Child PTSD Symptom Scale in Nepal. BMC Psychiatry 2011, 11(1):e127.

21. Kaiser BN, Kohrt BA, Keys HM, Khoury NM, Brewster A-RT: Strategies for assessing mental health in Haiti: Local instrument development and transcultural translation. Transcultural Psychiatry 2013, 50(4):532-558.

22. Kaiser BN, Ticao C, Anoje C, Minto J, Boglosa J, Kohrt BA: Adapting culturally appropriate mental health screening tools for use among conflict-affected and other vulnerable adolescents in Nigeria. Global mental health (Cambridge, England) 2019, 6:e10.

23. Rowntree O, Shanahan M: Connected Women: The Mobile Gender Gap Report 2020. In.: GSMA; 2020: 52.

24. Cornet VP, Holden RJ: Systematic review of smartphone-based passive sensing for health and wellbeing. J Biomed Inform 2018, 77:120-132.

25. Kohrt BA, Rai S, Vilakazi K, Thapa K, Bhardwaj A, van Heerden A: Procedures to Select Digital Sensing Technologies for Passive Data Collection With Children and Their Caregivers: Qualitative Cultural Assessment in South Africa and Nepal. JMIR Pediatr Parent 2019, 2(1):e12366. 
26. Slater H, Campbell JM, Stinson JN, Burley MM, Briggs AM: End User and Implementer Experiences of mHealth Technologies for Noncommunicable Chronic Disease Management in Young Adults: Systematic Review. J Med Internet Res 2017, 19(12):e406.

27. Poudyal A, van Heerden A, Hagaman A, Maharjan SM, Byanjankar P, Subba P, Kohrt BA: Wearable Digital Sensors to Identify Risks of Postpartum Depression and Personalize Psychological Treatment for Adolescent Mothers: Protocol for a Mixed Methods Exploratory Study in Rural Nepal. JMIR Res Protoc 2019, 8(8):e14734.

28. Eysenbach G: CONSORT-EHEALTH: Improving and Standardizing Evaluation Reports of Web-based and Mobile Health Interventions. J Med Internet Res 2011, 13(4):e126.

29. Gaglio B, Shoup JA, Glasgow RE: The RE-AIM Framework: A Systematic Review of Use Over Time. American Journal of Public Health 2013, 103(6):e38-e46.

30. United Nations Statistics Division: National Population and Housing Census 2011: National Report. In.; 2011.

31. Jordans MJD, Luitel NP, Garman E, Kohrt BA, Rathod SD, Shrestha P, Komproe IH, Lund C, Patel V: Effectiveness of psychological treatments for depression and alcohol use disorder delivered by community-based counsellors: two pragmatic randomised controlled trials within primary healthcare in Nepal. Br J Psychiatry 2019, 215(2):485-493.

32. Youth [https://www.un.org/en/sections/issues-depth/youth-0/index.html]

33. Hagaman AK, Khadka S, Lohani S, Kohrt B: Suicide in Nepal: a modified psychological autopsy investigation from randomly selected police cases between 2013 and 2015. Social Psychiatry and Psychiatric Epidemiology 2017, 52(12):1483-1494.

34. Leon AC, Davis LL, Kraemer HC: The role and interpretation of pilot studies in clinical research. Journal of psychiatric research 2011, 45(5):626-629.

35. Thabane L, Ma J, Chu R, Cheng J, Ismaila A, Rios LP, Robson R, Thabane M, Giangregorio L, Goldsmith $\mathrm{CH}$ : A tutorial on pilot studies: the what, why and how. BMC Medical Research Methodology 2010, 10(1):1-10.

36. Radius Networks Store: RadBeacon. In.; 2019.

37. van Heerden A, Wassenaar D, Essack Z, Vilakazi K, Kohrt BA: In-Home Passive Sensor Data Collection and Its Implications for Social Media Research: Perspectives of Community Women in Rural South Africa. Journal of Empirical Research on Human Research Ethics 2020, 15(1-2):97-107.

38. Acharya B, Basnet M, Rimal P, Citrin D, Hirachan S, Swar S, Thapa P, Pandit J, Pokharel R, Kohrt B: Translating mental health diagnostic and symptom terminology to train health workers and engage patients in cross-cultural, non-English speaking populations. International Journal of Mental Health Systems 2017, 11(1):62.

39. Tong A, Sainsbury P, Craig J: Consolidated criteria for reporting qualitative research (COREQ): a 32item checklist for interviews and focus groups. International Journal for Quality in Health Care 2007, 19(6):349-357. 
40. Jiao L, Zhang F, Liu F, Yang S, Li L, Feng Z, Qu R: A Survey of Deep Learning-Based Object Detection. IEEE Access 2019, 7:128837-128868.

41. Abeßer J: A review of deep learning based methods for acoustic scene classification. Applied Sciences 2020, 10(6).

42. QSR International: NVIVO qualitative data analysis software. In., 10 edn. Doncaster, Australia: QSR International Pty Ltd.; 2012.

43. Bernard HR: Analyzing Qualitative Data: Systematic Approaches, vol. 2nd: SAGE Publications Inc; 2016.

44. Guest GS: Applied thematic analysis, vol. 1st edition: SAGE Publications, Inc; 2011.

45. Woodall A, Morgan C, Sloan C, Howard L: Barriers to participation in mental health research: are there specific gender, ethnicity and age related barriers?BMC Psychiatry 2010, 10:103.

46. Jorgensen R, Munk-Jorgensen P, Lysaker PH, Buck KD, Hansson L, Zoffmann V: Overcoming recruitment barriers revealed high readiness to participate and low dropout rate among people with schizophrenia in a randomized controlled trial testing the effect of a Guided Self-Determination intervention. BMC Psychiatry 2014, 14:28.

47. Kannisto KA, Korhonen J, Adams CE, Koivunen MH, Vahlberg T, Valimaki MA: Factors Associated With Dropout During Recruitment and Follow-Up Periods of a mHealth-Based Randomized Controlled Trial for Mobile.Net to Encourage Treatment Adherence for People With Serious Mental Health Problems. J Med Internet Res 2017, 19(2):e46.

48. Kumar D, Tully LM, losif AM, Zakskorn LN, Nye KE, Zia A, Niendam TA: A Mobile Health Platform for Clinical Monitoring in Early Psychosis: Implementation in Community-Based Outpatient Early Psychosis Care. JMIR Ment Health 2018, 5(1):e15.

49. Kotz D, Gunter CA, Kumar S, Weiner JP: Privacy and Security in Mobile Health: A Research Agenda. Computer (Long Beach Calif) 2016, 49(6):22-30.

50. Abdullah S, Matthews M, Frank E, Doherty G, Gay G, Choudhury T: Automatic detection of social rhythms in bipolar disorder. J Am Med Inform Assoc 2016, 23(3):538-543.

51. Ben-Zeev D, Schueller SM, Begale M, Duffecy J, Kane JM, Mohr DC: Strategies for mHealth research: lessons from 3 mobile intervention studies. Adm Policy Ment Health 2015, 42(2):157-167.

52. Dewa LH, Lavelle M, Pickles K, Kalorkoti C, Jaques J, Pappa S, Aylin P: Young adults' perceptions of using wearables, social media and other technologies to detect worsening mental health: A qualitative study. PLoS One 2019, 14(9):e0222655.

53. Arsand E, Muzny M, Bradway M, Muzik J, Hartvigsen G: Performance of the first combined smartwatch and smartphone diabetes diary application study. J Diabetes Sci Techno/2015, 9(3):556-563.

54. Patel V, Weobong B, Weiss HA, Anand A, Bhat B, Katti B, Dimidjian S, Araya R, Hollon SD, King M et al: The Healthy Activity Program (HAP), a lay counsellor-delivered brief psychological treatment for severe depression, in primary care in India: a randomised controlled trial. Lancet 2017, 389(10065):176-185. 
55. Byanjankar P, Poudyal A, Kohrt B, Maharjan S, Hagaman A, van Heerden A: Utilizing passive sensing data to provide personalized psychological care in low-resource settings [version 1; peer review: awaiting peer review]. Gates Open Research 2020, 4(118).

\section{Tables}

Table 1: Demographic characteristics of study sample $(n=38)$ 


\begin{tabular}{|c|c|}
\hline Characteristics & N (\%) \\
\hline Mother's mental health status & $38(100)$ \\
\hline Depressed & $11(28.9)$ \\
\hline Non-depressed & $27(71.1)$ \\
\hline Mother's age & $\mathbf{N}(\%)$ \\
\hline $15-18$ years & $11(28.9)$ \\
\hline $19-22$ years & $22(57.9)$ \\
\hline 23-25 years & $5(13.2)$ \\
\hline Mother's caste/ethnicity & $\mathbf{N}(\%)$ \\
\hline Brahman/Chhetri (upper castes) & $8(21.0)$ \\
\hline Janajati (ethnic minorities) & $18(47.5)$ \\
\hline Dalit (lower castes) & $12(31.5)$ \\
\hline Mother's religion & $\mathbf{N}(\%)$ \\
\hline Hindu & $32(84.2)$ \\
\hline Buddhist & $3(7.9)$ \\
\hline Christian & $3(7.9)$ \\
\hline Mother's education & $\mathbf{N}(\%)$ \\
\hline Grades 1-5 & $7(18.4)$ \\
\hline Grades 6-10 & $24(63.2)$ \\
\hline Grades 11-12 & $7(18.4)$ \\
\hline Mother's Occupation & $\mathbf{N}(\%)$ \\
\hline Business & $3(7.9)$ \\
\hline Housewife & $29(76.3)$ \\
\hline Agriculture & $4(10.5)$ \\
\hline Day wage laborer & $2(5.3)$ \\
\hline Mother's number of children & N (\%) \\
\hline One child & $31(81.6)$ \\
\hline More than one child & $7(18.4)$ \\
\hline Mother's smartphone ownership & N (\%) \\
\hline Yes & $26(68.4)$ \\
\hline No & $12(31.6)$ \\
\hline
\end{tabular}




\begin{tabular}{|l|l|} 
Infant's gender & $\mathbf{N}(\%)$ \\
\hline Male & $16(42.1)$ \\
\hline Female & $22(57.9)$ \\
\hline Infant's age & $\mathbf{N}(\%)$ \\
\hline 1 to 4 months & $21(55.2)$ \\
\hline 5 to 8 months & $10(26.3)$ \\
\hline 9 to 12 months & $7(18.5)$ \\
\hline Household: Electricity & $\mathbf{N}(\%)$ \\
\hline Yes & $36(94.7)$ \\
\hline No & $2(5.3)$ \\
\hline Household: WiFi internet at home & $\mathbf{N}(\%)$ \\
\hline Yes & $3(7.9)$ \\
\hline No & $35(92.1)$ \\
\hline
\end{tabular}

Table 2: Total passive data collected daily from 4 AM to 9 PM 


\begin{tabular}{|c|c|c|c|c|c|}
\hline $\begin{array}{l}\text { Passive } \\
\text { sensing } \\
\text { domain }\end{array}$ & $\begin{array}{l}\text { Total possible } \\
\text { number of } \\
\text { readings }\end{array}$ & $\begin{array}{l}\text { Observed } \\
\text { number of } \\
\text { readings }\end{array}$ & $\begin{array}{l}\text { Percent mean observed } \\
\text { readings per } \\
\text { participant }\end{array}$ & $\begin{array}{l}\text { Range of percent } \\
\text { readings (min- } \\
\text { max) }\end{array}$ & $\begin{array}{l}\text { Median } \\
\text { percent } \\
\text { readings (IQR) }\end{array}$ \\
\hline \multicolumn{6}{|c|}{ All participants $(n=38)$} \\
\hline Audio & 9,605 & 5579 & $57.4 \%$ & 11.7- $97.2 \%$ & $62.6 \%(25.1)$ \\
\hline Activity & 9,605 & 5001 & $50.6 \%$ & $0-95.5 \%$ & $63.2 \%(51.1)$ \\
\hline Proximity & 9,605 & 4168 & $41.1 \%$ & $0-84.2 \%$ & $47.6 \%(32.5)$ \\
\hline GPS & 9,605 & 3482 & $35.4 \%$ & $0-85.3 \%$ & $39.2 \%(45.4)$ \\
\hline \multicolumn{6}{|c|}{ Non-depressed participants $(n=27)$} \\
\hline Audio & 6,902 & 3,926 & 57.8 & $13.2-87.9$ & 63.7 (17.5) \\
\hline Activity & 6,902 & 3,304 & 48.1 & $0-87.9$ & $62.4(53)$ \\
\hline Proximity & 6,902 & 3,087 & 43.9 & $1-84.2$ & $51.8(35.4)$ \\
\hline GPS & 6,902 & 2,527 & 36.7 & $0-85.3$ & $40.8(47.9)$ \\
\hline \multicolumn{6}{|c|}{ Depressed participants $(n=11)$} \\
\hline Audio & 2,703 & 1,653 & 56.7 & 11.8-97.1 & 51.2 (31.9) \\
\hline Activity & 2,703 & 1,697 & 56.9 & $0-95.5$ & $64.0(28.1)$ \\
\hline Proximity & 2,703 & 1,081 & 34.0 & $0-67$ & 32.9 (20.8) \\
\hline GPS & 2,703 & 955 & 32.4 & $0-77.5$ & 30.4 (39.9) \\
\hline
\end{tabular}

\section{Table 3: Qualitative Domains}




\begin{tabular}{|l|l|l|}
\hline Domains & Definition & Code Examples \\
\hline $\begin{array}{l}\text { Domain 1: } \\
\text { Technical } \\
\text { issues }\end{array}$ & $\begin{array}{l}\text { The degree to which technical issues in the } \\
\text { devices impact use or the devices. }\end{array}$ & $\begin{array}{l}\text { mobile use, data } \\
\text { usage, family } \\
\text { involvement }\end{array}$ \\
\hline $\begin{array}{l}\text { Domain 2: } \\
\text { Interference }\end{array}$ & $\begin{array}{l}\text { The degree to which the device may impact } \\
\text { physical functioning, activities, or daily routines. }\end{array}$ & $\begin{array}{l}\text { mobile use, battery, } \\
\text { beacon use }\end{array}$ \\
\hline $\begin{array}{l}\text { Domain 3: } \\
\text { Confidentiality }\end{array}$ & $\begin{array}{l}\text { The degree to which the device would protect } \\
\text { personal information. }\end{array}$ & $\begin{array}{l}\text { privacy concerns, } \\
\text { social perspective }\end{array}$ \\
\hline $\begin{array}{l}\text { Domain 4: } \\
\text { Safety }\end{array}$ & $\begin{array}{l}\text { Perceptions regarding health risks or put a child, } \\
\text { mother or family at the risk of mugging or theft. } \\
\text { This domain also explores safety concerns } \\
\text { mothers have over losing or breaking the device } \\
\text { itself. }\end{array}$ & $\begin{array}{l}\text { child safety, mother } \\
\text { safety, device safety }\end{array}$ \\
\hline $\begin{array}{l}\text { Domain 5: } \\
\text { Utility }\end{array}$ & $\begin{array}{l}\text { The perceived benefits of the device for } \\
\text { improving caregiver and child health, } \\
\text { development, and mental health. }\end{array}$ & $\begin{array}{l}\text { non-study specific } \\
\text { utility, study specific } \\
\text { utility, } \\
\text { misperceptions and } \\
\text { other perceptions }\end{array}$ \\
\hline $\begin{array}{l}\text { Domain 6: } \\
\text { Communication }\end{array}$ & $\begin{array}{l}\text { Communicating study objectives and device use } \\
\text { to the mothers during the consent process at the } \\
\text { beginning of the study and by study team } \\
\text { engagement throughout the study duration. }\end{array}$ & $\begin{array}{l}\text { autonomy, study } \\
\text { team engagement, } \\
\text { consent/debriefing } \\
\text { process }\end{array}$ \\
\hline
\end{tabular}

\section{Figures}


a.

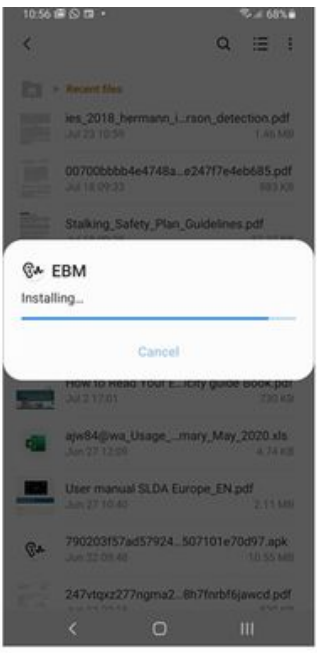

c.
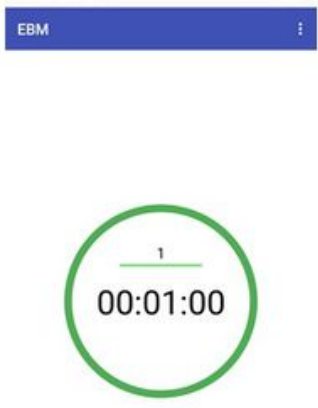

-

e.

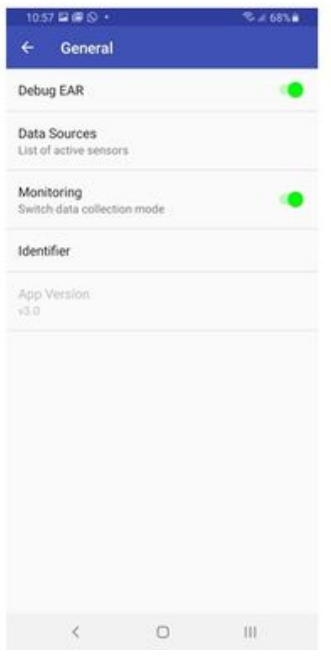

b.

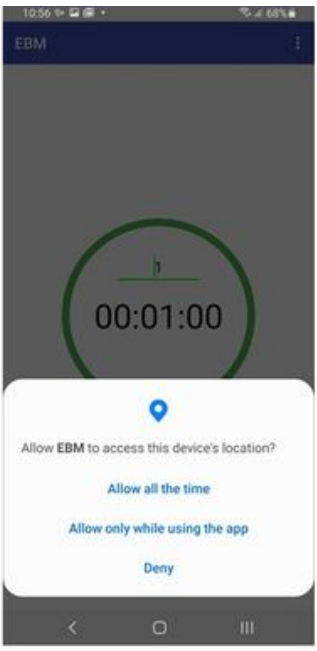

d.

Евм

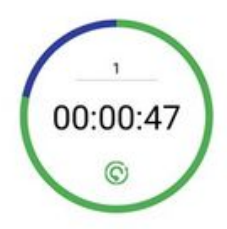

(1)

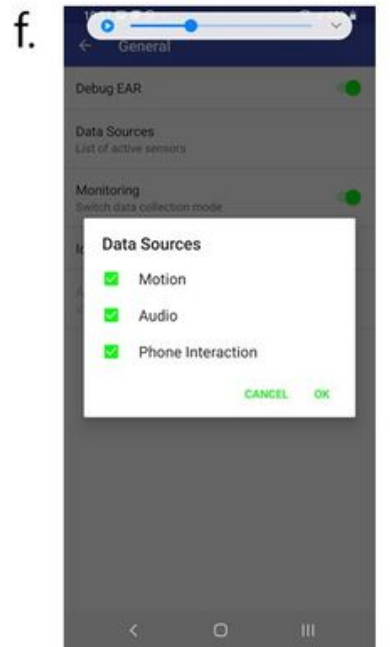

Figure 1

Screenshots of Electronic Behavior Monitoring app (EBM version 2.0). a. EBM package installer; b. EBM permission controller; c. EBM privacy timer; d. EBM privacy timer running; e. EBM settings; f. EBM settings sources. 
$100 \%$

$90 \%$

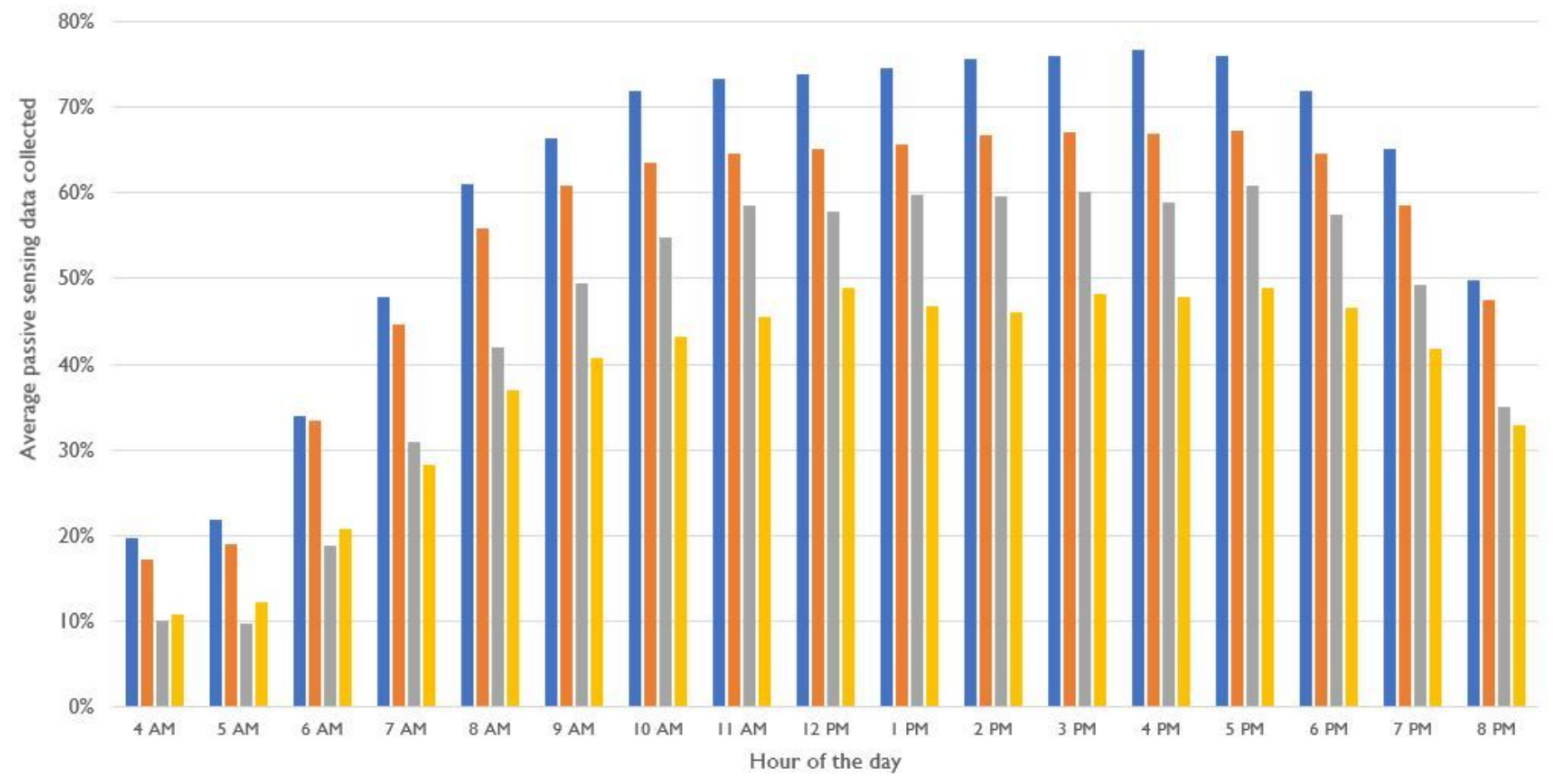

- Audio recordings

In Physical activity readings

Mother-infant proximity

GPS location

\section{Figure 2}

Average passive data collection by the time of day, based on readings collected from 4 AM to 9 PM for two weeks with depressed and non-depressed mothers.

\section{Supplementary Files}

This is a list of supplementary files associated with this preprint. Click to download.

- PublicationFilessupplementalonlinefile.docx

- SupplementalFile1EHEALTHCONSORTCHECKLIST.docx

- SupplementalFile2REAIMFramework.docx

- SupplementalFile4SupplementalTables.docx

- SupplementalFile3COREQQualitativeReportingChecklist.docx 